\title{
La Structure Du Pouvoir Judiciaire au Brésil: Des Poursuites Ciminelles Aux Récentes Réformes Dans Le Code de Procédure Pénale Brésilien
}

\author{
The Structure of Judiciary in Brazil: From Criminal Prosecution \\ To Recent Reforms in Brazilian Criminal Procedure Code
}

Tarsis Barreto Oliveira ${ }^{1}$

\section{RÉSUMÉ:}

L'article aborde une brève vision de la structure du Pouvoir judiciaire au Brésil, circonscrite par la Constitution de 1988, et qui s'attache notamment à la structure juridictionnelle pénale. Tout d'abord il est procédé à l'examen des organes qui composent la justice brésilienne, leurs attributions et compétences, avant de passer aux fonctions essentielles à la justice. Il est question ensuite des types de peines admissibles et les règles de leur application. On procède enfin à l'examen des poursuites pénales engagées au Brésil, qu'il s'agisse d'enquêtes criminelles, d'arrestations ou de poursuites pénales, ou encore des récentes réformes introduites par le législateur dans le Code de procédure pénale.

MOTS-CLÉS:

Pouvoir judiciaire; poursuites pénales; peines; réformes; code de procédure pénale.

\begin{abstract}
:
This article presents a brief analysis on the structure of the Judiciary in Brazil, delimited by the Constitution of 1988, with special emphasis on the penal jurisdictional structure. Initially we analyse the organs that compose Brazilian Justice, its attributions and competences, and then the essential functions to Justice. Then we examine the kinds of penalties allowed and the rules for their enforcement. Finally we examine criminal prosecution in Brazil, starting from criminal investigation, kinds of arrest and criminal prosecution, to the recent reforms introduced by the legislator in the Criminal Procedure Code.
\end{abstract}

KEYWORDS:

Judiciary; Criminal prosecution; Penalties; Reforms; Criminal Procedure Code.

\footnotetext{
${ }^{1}$ Docteur en Droit de l'Université Fédérale de Bahia (Brésil). Professeur de Droit Pénal de l'Université Fédérale de Tocantins. Coordinateur du Cours de Spécialisation en Sciences Criminelles de la UFT. Membre du Comité International des Pénalistes Francophones et de l'Association Internacionale de Droit Pénal.
} 


\section{STRUCTURE DU POUVOIR JUDICIAIRE BRÉSILIEN}

Le Pouvoir judiciaire brésilien est structuré dans les organes suivants: a) Cour suprême fédérale; b) Conseil national de justice; c) Cour supérieure de justice; d) Tribunaux fédéraux régionaux et juges fédéraux; e) Tribunaux et juges du travail; f) Tribunaux et juges électoraux; g) Tribunaux et juges militaires; h) Tribunaux et juges des États, du District fédéral et des Territoires.

La Cour suprême fédérale ${ }^{2}$, la plus haute cour de justice au Brésil, est composée de onze ministres, citoyens de plus de trente-cinq ans et de moins de soixante-cinq ans ayant des connaissances juridiques notoires et une réputation irréprochable. Ils sont choisis par le Président de la République après approbation par la majorité absolue du Sénat fédéral.

La Cour suprême fédérale a pour mission d'être le gardien de la Constitution, se devant de poursuivre et de juger: a) les questions prioritaires de constitutionnalité d'une loi ou d'un acte réglementaire fédéral ou d'un État et l'action déclaratoire de constitutionnalité d'une loi ou d'un acte réglementaire fédéral; b) pour les infractions de droit commun, le Président de la République, le Vice-président de la République, les membres du Congrès national, les Ministres d'État et le Procureur général de la République; c) pour les infractions de droit commun et les « crimes de responsabilité », les Ministres d'État et les Commandants de la Marine, de l'Armée de terre et de l'Armée de l'air; d) les procédures impliquant le Brésil et d'autres pays et organisations internationales; e) les procédures d'extradition; f) les conflits de compétence la concernant; g) les crimes politiques; h) la constitutionnalité des lois et actes réglementaires; entre autres attributions.

Nous avons ensuite le Conseil national de justice. Il est composé de 15 membres avec un mandat de 2 ans et il est présidé par le Président de la Cour suprême fédérale. Comme le

\footnotetext{
${ }^{2}$ Selon Lenza (2007, p. 525), au cours du régime monarchique, la loi du 18/09/1828 a créé la dénommée Cour suprême de justice. Le décret n 848 du 11/10/1890, édicté par le gouvernement provisoire de la République, a organisé la Cour suprême fédérale, organe supérieur de la justice conformément aux articles 55 et 56 de la Constitution républicaine de 1891, effectivement installée le 28/02/1891.
} 
rappelle Cunha Júnior (2008, p. 933), le Conseil national de justice, bien qu'il intègre la structure du pouvoir judiciaire, ne dispose pas de fonction juridictionnelle.

Sa mission est de contrôler les activités administratives et financières de la magistrature, ainsi que l'exercice des fonctions officielles des juges, mais aussi de: a) garantir l'indépendance du pouvoir judiciaire et le respect du Statut des magistrats; b) superviser les actes administratifs réalisés par les membres de la magistrature; c) recevoir, analyser et juger les plaintes contre les membres ou organes judiciaires; d) communiquer au ministère public les cas de crime contre l'administration publique ou les abus de pouvoir; e) élaborer des rapports sur les procédures et les sentences proférées par la magistrature; f) etc.

La Cour supérieure de justice est composée au moins de trente-trois ministres, nommés par le Président de la République, âgés d'au moins trente-cinq ans et au plus de soixante-cinq ans, aux connaissances juridiques notoires et à la réputation irréprochable, après approbation du choix à la majorité absolue du Sénat fédéral. Entre autres attributions elle a pour fonction de poursuivre et de juger: a) pour les crimes de droit commun, les Gouverneurs des États et du District fédéral et pour ceux-ci et pour les «crimes de responsabilité », les juges de seconde instance des Tribunaux de justice des États et du District fédéral, les membres des Cours des comptes des États et du District fédéral, des Tribunaux fédéraux régionaux, des Tribunaux électoraux régionaux et du Travail, les membres des Conseils ou des Chambres régionales des comptes et ceux du Ministère public fédéral qui officient dans les tribunaux; b) les injonctions interlocutoires et les habeas data contre des actes d'un Ministre d'État, des Commandants de la Marine, de l'Armée de terre et de l'Armée de l'air ou du tribunal lui-même; c) les conflits de compétence entre les tribunaux; d) les conflits d'attributions entre les autorités administratives et judiciaires du Gouvernement fédéral ou entre les autorités judiciaires d'un État et administratives d'un autre État ou du District fédéral, ou entre celles du District fédéral et celles du Gouvernement fédéral; e) les cas où les parties sont un État étranger ou une organisation internationale, d'une part, et d'autre part, une municipalité ou une personne résidant ou domiciliée dans le pays; etc.

Les Tribunaux fédéraux régionaux sont composés d'au moins sept juges, choisis, chaque fois que possible, dans la région respective et nommés par le Président de la République parmi les Brésiliens de plus de trente ans et de moins de soixante-cinq ans. Ils sont en droit de 
poursuivre et de juger, initialement: a) les juges fédéraux de la région de leur compétence, y compris ceux de la Justice militaire et ceux des Tribunaux du travail, en droit commun et «crime de responsabilité», et les procureurs du Ministère Public du Gouvernement fédéral; b) les révisions pénales et les actions pénales de rétractation contre leurs décisions ou de celles des juges fédéraux de la région; c) les injonctions interlocutoires et les habeas data contre un acte du Tribunal lui-même ou d'un juge fédéral; d) les habeas corpus, lorsque l'autorité contraignante est un juge fédéral; e) les conflits de compétence entre les juges fédéraux assujettis au Tribunal; f) juger en appel les affaires tranchées par les juges fédéraux et par les juges de l'État dans l'exercice de la compétence fédérale dans leur domaine de juridiction.

Quant aux juges fédéraux ils ont comme compétence de juger: a) les cas dans lesquels le Gouvernement fédéral, une agence gouvernementale autonome ou une société publique fédérale sont impliqués en tant que demandeurs, défendeurs, ayants droit ou opposants à l'exception des cas de faillite, des accidents de travail et des cas soumis aux tribunaux électoraux et à ceux du travail; b) les cas entre un État étranger ou une organisation internationale et une municipalité ou une personne domiciliée ou résidant dans le pays; c) les cas fondés sur un traité ou un accord du Gouvernement fédéral avec un État étranger ou une organisation internationale; d) les crimes politiques et les infractions commises contre les biens, les services ou les intérêts du Gouvernement fédéral ou de ses organismes autonomes ou entreprises publiques; e) les crimes prévus dans les traités ou conventions internationales; f) les cas relatifs aux droits de l'homme; g) les crimes contre l'organisation du travail, contre le système financier et l'ordre économique et financier; h) les habeas corpus, en matière pénale relevant de sa compétence; i) les injonctions interlocutoires et les habeas data contre un acte d'une autorité fédérale; j) les crimes commis à bord de navires ou d'aéronefs; entre autres fonctions.

La Justice du travail, elle, comprend les organes suivants: la Cour supérieure du travail (formée de 27 ministres aux connaissances juridiques notoires et à la réputation irréprochable, avec la fonction de juger, entre autres questions, les actions liées au droit de grève, impliquant les syndicats et les travailleurs); les Tribunaux régionaux du travail (composés d'au moins 7 juges), et les juges du travail. Les deux ont pour attribution de juger les actions provenant des relations de travail, dans les limites de leurs compétences. 
La Justice électorale est composée des organes suivants: la Cour supérieure électorale (composée de sept membres); les Tribunaux électoraux régionaux; les Juges électoraux; les conseils électoraux. Les deux ont la tâche de juger les actions du processus électoral brésilien.

En ce qui concerne les organes de justice militaire, ceux-ci sont divisés en: Cour militaire supérieure (composée de 15 ministres à vie); les Tribunaux militaires; et les juges militaires, les deux ayant pour attribution de juger les crimes militaires définis dans la loi, dans les limites de leurs compétences.

Enfin, nous avons les tribunaux de justice et les juges des États qui jugent les autres questions, en respectant les limites de leurs compétences et de leurs obligations légales.

\section{DES FONCTIONS ESSENTIELLES À LA JUSTICE}

Le Ministère Public est au Brésil une institution permanente, essentielle à la fonction juridictionnelle de l'État, qui a la tâche de défendre l'ordre juridique, le régime démocratique et les intérêts sociaux et individuels inaliénables; c'est un corps doté d'une autonomie fonctionnelle et administrative et il comporte: a) le Ministère public fédéral; b) le Ministère public du travail; c) le Ministère public militaire; d) le Ministère public du District fédéral et des Territoires; e) les Ministères publics des États.

La Constitution fédérale de 1988 a donné au Ministère public brésilien de nouvelles dimensions, en le plaçant dans une position d'égalité avec les Pouvoirs de la République et en lui assignant plusieurs fonctions institutionnelles, ainsi qu'en lui fournissant les garanties juridiques et les instruments nécessaires à la réalisation de ses objectifs. (FREITAS; SOUZA, 2013, p. 77)

Le Ministère public a les fonctions institutionnelles suivantes: promouvoir, en exclusivité, l'action pénale publique, conformément à la loi; s'assurer que les pouvoirs publics respectent effectivement les droits garantis par la Constitution, en prenant les mesures nécessaires pour les respecter; instituer l'enquête civile et l'action civile publique pour protéger 
la propriété publique et sociale, l'environnement et d'autres intérêts diffus et collectifs; judiciairement, défendre les droits et les intérêts des peuples autochtones; exercer un contrôle externe sur les activités de la police, demander des procédures d'enquête et l'institution des enquêtes de police, entre autres fonctions.

Enfin, la Défense des intérêts publics est exercée au niveau fédéral et des États. Au niveau fédéral, il y a la Défense générale du Gouvernement fédéral, dont la mission est de le représenter, judiciairement et extrajudiciairement. Au niveau des États, on trouve la Défense générale de chaque État, dont la mission est de représenter les États, judiciairement et extrajudiciairement.

Guimarães (2011, p. 10) signale que la création d'une institution spécialisée responsable de toutes les activités de contentieux et de conseil représente l'option spécifique de certains pays, tels que le Brésil, l'Italie et l'Espagne, visant à attribuer la protection judiciaire et le conseil juridique de l'Etat à un seul organe, pour des raisons d'efficacité et d'uniformité.

Il convient de noter aussi le Corps des défenseurs publics, présent dans tous les états de la fédération, considéré par la Constitution comme une institution permanente, essentielle à la fonction juridictionnelle de l'État, avec pour mission le conseil juridique, la promotion des droits de l'homme et la protection dans toutes les instances des droits judiciaires et extrajudiciaires, individuels et collectifs, des nécessiteux de manière complète et gratuite, à condition que soit reconnue leur situation.

Notons également que la Constitution brésilienne reconnaît le rôle important du droit privé, considérant qu'il est essentiel à l'administration de la justice; les actes ou les manifestations de l'avocat, dans l'exercice de sa profession et dans les limites de la loi, sont marquées par l'inviolabilité. 


\section{LES PEINES DANS LE DROIT BRÉSILIEN}

Au Brésil sont recevables 3 espèces de peines: a) la privation de liberté; b) la restriction des droits; c) l'amende. À cet égard, la Constitution fédérale interdit expressément, à l'article 5, paragraphe XLVII, l'adoption dans notre ordonnancement de la peine de mort (sauf en cas de guerre déclarée); l'emprisonnement à vie; le travail forcé; le bannissement; et les actes cruels ${ }^{3}$.

Les peines privatives de liberté sont divisées en deux espèces: Réclusion et Détention. La différence entre elles est que la peine de Réclusion est purgée selon trois régimes: a) fermé (avec exécution de la peine dans un établissement de sécurité maximale ou moyenne), semiouvert (avec l'exécution de la peine en colonie agricole, industrielle ou dans un établissement similaire) et ouvert (exécution de la peine dans un centre de semi-liberté ou dans un établissement approprié). Quant à la peine de détention elle admet les deux derniers régimes: semi-ouvert ou ouvert.

Ainsi, l'exécution de la peine au Brésil se fait progressivement, à savoir les condamnés commencent à purger leur peine dans un régime strict et peu à peu, ayant satisfait aux exigences légales (de nature objective et subjective), on en arrive à l'accomplissement de la peine dans un régime plus favorable.

La détermination du début de l'accomplissement de la peine doit respecter les règles suivantes: a) le condamné à plus de huit ans doit commencer à l'accomplir dans un régime fermé; b) le condamné non-récidiviste dont la peine est de plus de 4 ans et ne dépassant pas huit ans, peut, dès le début, l'accomplir dans le régime semi-ouvert; c) le condamné non-récidiviste, dont la peine est égale ou inférieure à 4 ans, peut, dès le départ, l'accomplir dans un régime ouvert.

Dans le système fermé, le condamné s'adonne au travail pendant la journée et est soumis à l'isolement pendant la nuit. Le travail n'est pas obligatoire dans les prisons

\footnotetext{
${ }^{3}$ À cet égard, il convient de rappeler que le Code pénal brésilien a adopté la théorie dite mixte ou éclectique de la peine, selon laquelle les objectifs de la peine sont la rétribution, la prévention et la resocialisation. (OLIVEIRA, 2017, p. 150).
} 
brésiliennes (étant donné que le travail forcé n'est pas permis) ${ }^{4}$, mais le condamné qui travaille a droit au bénéfice de réduction de peine, de sorte que trois jours de travail donnent droit à 1 jour de peine en moins. Le travail doit aussi respecter les compétences ou les occupations antérieures du condamné, et doit être payé par l'État, les prestations de la Sécurité sociale devant être garanties au prisonnier.

Dans le régime semi-ouvert, le condamné est soumis à un travail en commun pendant la journée dans la colonie agricole, industrielle ou dans un établissement similaire. Dans ce régime, le condamné est autorisé à travailler hors de l'établissement pénitentiaire, ainsi qu'à suivre des cours de professionnalisation, des études secondaires ou supérieures.

Quant au régime ouvert il est basé sur l'autodiscipline et le sens de la responsabilité du condamné, car celui-ci doit travailler hors de l'établissement sans surveillance, assister à un cours ou exercer une autre activité autorisée, étant reclus pendant la nuit et les jours de congé.

Comme le souligne Greco (2014, pp. 503-504), la particularité du régime ouvert par rapport aux régimes précédents concerne le travail, puisque dans les régimes fermés et semiouverts, le travail du détenu lui donne droit à une remise; quant au régime ouvert, il n'y a pas de disposition légale de remise de peine pour travail, puisque seule la personne condamnée qui travaille ou prouvera la possibilité de le faire pourra y prétendre immédiatement.

Si le prisonnier ne se conforme pas à l'une des conditions imposées dans un régime plus favorable, a lieu ce que l'on appelle la régression, il retourne dans un régime plus strict pour accomplir sa peine.

Selon le Code pénal brésilien le prisonnier conserve tous les droits qui ne sont pas affectés par la perte de la liberté, les autorités devant respecter son intégrité physique et morale, ce qui, cependant, n'a pas empêché au Brésil l'apparition de nombreux cas de torture et de violence contre les détenus.

Le deuxième type de sanction en Droit pénal brésilien correspond à des sanctions limitant les droits. Elles sont appliquées pour remplacer les peines privatives de liberté jusqu'à

\footnotetext{
${ }^{4}$ Selon Coêlho (2014, p.305), puisque le prisonnier a le droit de travailler, si cela lui est refusé, l'Etat doit obligatoirement supporter le bénéfice de réduction de peine pour les jours non travaillés, comme s'ils avaient été effectués, conformément à l'Article 126 de la loi brésilienne d'exécution pénale.
} 
la limite de 4 ans, à condition que le condamné ne soit pas un récidiviste en infraction intentionnelle, que le crime n'ait pas été commis avec violence ou menace grave, et que la culpabilité, les antécédents, le comportement social, la personnalité, les motivations et les circonstances du condamné indiquent que la substitution par une peine alternative est appropriée et suffisante.

Les peines restrictives de droits admettent les formes suivantes: a) des prestations en espèces (paiement en espèces à la victime, aux personnes à sa charge ou à une entité publique ou privée de caractère social); b) la perte de biens et avoirs (appartenant au condamné à la suite de la pratique du crime); c) la prestation de services à la communauté ou à des entités publiques (obligation faite au condamné de fournir des services libres dans des organismes de bienfaisance, des hôpitaux, des écoles, des orphelinats et autres établissements similaires); d) la suspension temporaire des droits (dont les modalités sont les suivantes: interdiction d'exercice de fonction, d'activité publique ainsi que de mandat électif; interdiction d'exercer la profession, l'activité ou le métier qui repose sur une licence spéciale, un permis ou une autorisation du gouvernement; suspension de l'autorisation ou du permis de conduire des véhicules; l'interdiction de fréquenter certains lieux); e) la limitation du week-end (obligation de demeurer, les samedis et dimanches, pendant 5 heures par jour dans un centre de semi-liberté ou dans un autre établissement approprié, où peuvent être donnés des cours et conférences au condamné, ou des activités éducatives lui être assignées).

La troisième modalité de peine autorisée en vertu de la loi brésilienne est la sanction de l'amende, consistant dans le paiement au FPN (Fonds pénitentiaire national) d'un montant fixé lors du jugement et calculé en amendes quotidiennes ${ }^{5}$, étant d'au moins 10 et au plus de 360 amendes quotidiennes. Le montant exact de l'amende quotidienne est fixé par le juge et ne peut pas être inférieur à un trentième du salaire minimum brésilien ni supérieur à 5 fois ce salaire.

Il convient également de noter que, conformément à l'article $75 \mathrm{du}$ Code pénal brésilien le temps d'emprisonnement au Brésil ne peut pas dépasser 30 ans.

\footnotetext{
${ }^{5}$ Selon la leçon d'Esfefam (2010, page 330), la réforme du Code pénal brésilien de 1984 a remis à l'honneur le système de l'amende-jour, révoquant toutes les dispositions du Code pénal et les lois spéciales prévoyant une sanction monétaire à taux fixe.
} 
Il y a quelques avantages pénaux appliqués aux condamnés au Brésil, comme le sursis (mise à l'épreuve) et la libération conditionnelle.

Grâce au sursis, l'exécution de la peine d'emprisonnement ne dépassant pas deux ans peut être suspendue pour une période de 2 à 4 ans (période de mise à l'épreuve), à condition que le condamné: ne soit pas un récidiviste d'un crime grave; que la culpabilité, les antécédents, le comportement social et la personnalité du détenu, ainsi que les raisons et les circonstances permettent l'octroi du bénéfice; qu'il ne soit pas approprié de le remplacer par une peine de restriction des droits.

Le sursis est appliqué à condition qu'il ne soit pas possible de substituer une privation de liberté à une sanction restrictive des droits (fixée pour une limite de 4 ans). Ainsi, considérant que le sursis est une institution subsidiaire à l'application d'une peine alternative, Capez (2012, p. 515) souligne, avec précision, qu'il n'y a que trois hypothèses pour appliquer le sursis au Brésil: a) les crimes intentionnels commis avec violence ou menace grave, pour lesquels la peine infligée est égale ou inférieure à deux ans; b) condamné qui récidive en commentant un crime intentionnel, dont la peine précédente était une amende; c) condamné qui récidive en commettant une infraction non intentionnelle.

Quant à la libération conditionnelle elle consiste dans l'anticipation de la mise en liberté du détenu condamné à moins de deux ans, à condition qu'il: a) ait purgé plus d'un tiers de la peine, ne soit pas récidiviste dans un crime grave et ait de bons antécédents; b) ait complété plus de la moitié de la peine, et soit récidiviste dans un crime grave, ait prouvé un comportement satisfaisant lors de l'accomplissement de la peine, de bons résultats dans le travail qui lui est attribué et la capacité de subvenir à ses besoins par un travail honnête; c) ait réparé, sauf si cela est impossible de le faire, les dommages causés par l'infraction; d) ait complété plus de deux tiers de la peine en cas de condamnation pour un crime odieux, la torture, le trafic illicite des stupéfiants et des drogues similaires, et le terrorisme, à condition qu'il ne soit pas récidiviste spécifique dans des crimes de cette nature.

Les personnes atteintes de maladie mentale ne sont l'objet de sanctions, mais au contraire, de mesures de sécurité, la personne devant être accueillie dans un établissement doté de caractéristiques hospitalières et soumise à un traitement. Les mesures de sécurité admettent 2 modes: I - hospitalisation en unité hospitalière spécialement aménagée et traitement 
psychiatrique ou, à défaut, dans une autre institution appropriée; II - soumis à un traitement ambulatoire. Contrairement à la peine, la mesure de sécurité n'a pas de date limite, elle est d'une durée indéterminée, jusqu'à ce que soit confirmée par un examen médical la fin de la dangerosité du détenu.

\section{LA POURSUUITE CRIMINELLE AU BRÉSIL}

L'enquête de police au Brésil est effectuée par la police, et elle a pour but d'investiguer les infractions pénales et leurs auteurs. Il est consensuel actuellement que le Ministère Public a, lui aussi, le pouvoir d'enquêter, de par le principe de la théorie des pouvoirs implicites. Cependant, il y n'y a pas encore de législation définissant les cas dans lesquels l'attribution reviendrait à cet organe, et comment, dans quel délai, et quelles en seraient les règles.

La date limite pour l'achèvement de l'enquête de police est de 10 jours (si l'accusé a été arrêté en flagrant délit, ou s'il est placé en détention provisoire) ou 30 jours (si l'accusé est libéré, sous caution ou non). Cette période peut être prolongée dans l'hypothèse où le prévenu est en liberté, si le commissaire de police prouve la complexité de l'enquête. Une fois l'enquête de police conclue, elle doit être portée devant la justice qui remettra les dossiers de l'enquête au Ministère Public, qui, à son tour, peut présenter une plainte contre l'accusé, ou alors classer sans suite la procédure. Toutefois, le Ministère Public peut demander la restitution de l'enquête à l'autorité policière afin qu'elle réalise d'autres investigations nécessaires à la présentation de la plainte.

C'est, en règle générale, le Ministère Public qui engage l'action pénale, en présentant une plainte dans les 5 jours (si l'accusé est arrêté) et 15 jours (si l'accusé est en liberté), délais courant à partir de la date de réception du dossier de l'enquête de police.

Selon Pacelli et Fischer (2013, p. 61), dans les poursuites publiques, le ministère public n'exerce pas un droit d'action, mais un devoir d'action, étant donné que le ministère public est soumis au principe de l'obligation. Ainsi, convaincu de l'existence d'un crime, ainsi que de sa 
paternité, et à condition qu'il examine les conditions de l'action pénale présente, le procureur doit soumettre l'affaire pénale à l'examen du pouvoir judiciaire.

Dans certains crimes spécifiques (comme les crimes contre l'honneur), c'est à la victime ou à son représentant légal qu'il revient de présenter la plainte. Dans ce cas, la date limite de présentation de la plainte est de 6 mois. Telles sont les hypothèses de l'action pénale privée. Selon Mirabete (2007, p. 106), bien que le droit de punir (jus puniendi) appartienne exclusivement à l'Etat, il transfère à l'individu le droit d'accuser (jus acusationis) dans certains cas, de telle manière que le droit de punir continue dans les mains de l'État, l'individu ayant le droit d'agir.

Dans son jugement, le juge doit fonder sa conviction sur la libre appréciation des preuves produites selon le principe du contradictoire et ne peut fonder sa décision uniquement sur les éléments d'information recueillis pendant l'enquête. Ne sont pas autorisées dans la procédure pénale brésilienne les preuves illicites, c'est-à-dire les preuves obtenues en violation des normes constitutionnelles et juridiques, et dans ce cas, elles doivent être retirées de la procédure, la nullité de l'acte de production des preuves pouvant être décrétée.

Quant à l'arrestation, elle est admise dans la procédure pénale brésilienne sous des espèces différentes. Initialement nous avons l'arrestation en flagrant délit ${ }^{6}$, qui est une mesure d'auto-défense de la société, caractérisée par une privation de liberté de mouvement de celui qui est surpris en situation de flagrance; elle doit être exécutée indépendamment d'une autorisation judiciaire préalable, et elle est considérée comme une forme d'ouverture de l'enquête de police. Notons que l'arrestation en flagrant délit peut devenir préventive, si sont présentes les exigences de l'article 312 du Code de procédure pénale ou si les diverses mesures de précaution de l'arrestation ne sont pas suffisantes.

Dans le contexte de l'arrestation en flagrant délit, un changement significatif est intervenu au Brésil à partir de 2015. La Cour suprême fédérale, compte tenu du chaos du système carcéral brésilien, a déterminé l'adoption de mesures immédiates pour la mise en œuvre

\footnotetext{
${ }^{6}$ Arrêter en flagrant délit est une mesure restrictive de liberté, de précaution et de procédure, consistant en l'arrestation de ceux qui sont surpris en train de commettre ou peu de temps après avoir commis une infraction pénale (crime ou contravention), ou aussi en possession d'instruments, arme et objets qui font présumer qu'il s'agit de l'auteur de l'infraction. (ISHIDA, 2009, p.161).
} 
par les juges et les tribunaux du pays des audiences de garde à vue. À cet égard, les audiences de garde à vue étaient déjà prévues dans le Pacte de San José, Costa Rica (accord international dont le Brésil est signataire), mais jusque-là n'avaient pas été mises en œuvre dans le pays. Dès l'année 2015, elles ont commencé à être mises en place dans tous les états de la fédération, jouant un rôle clé dans la mesure où elles servent de filtre constitutionnel pour la vérification des illégalités ou des abus dans les arrestations possibles, conduisant dans ce cas, à la remise en liberté de l'accusé (à condition que les mesures de protection soient respectées), moyennant l'utilisation du bracelet électronique à la cheville, et la responsabilité des fonctionnaires pour les excès éventuellement commis.

Un autre mode d'arrestation est l'arrestation temporaire. Celle-ci est appropriée: a) lorsqu'elle est nécessaire pour les investigations de l'enquête de police; b) si l'accusé n'a pas de résidence permanente ou ne fournit pas les informations nécessaires pour préciser son identité; c) quand il y a de bonnes raisons, conformément à toute preuve admise dans le droit pénal, que l'accusé a été auteur ou coauteur.

L'arrestation temporaire dure cinq jours et peut être prolongée pour la même période en cas de besoin avéré. Dans le cas d'un crime odieux, l'arrestation temporaire doit avoir une période de 30 jours et peut également être prolongée pour la même période en cas de besoin avéré.

Un autre mode d'arrestation est appelé détention provisoire en vertu des articles 311316 du Code de procédure pénale brésilienne. C'est une mesure de précaution de réclusion par laquelle le législateur répond à certains critères établis par la loi pour supputer entre le droit à la liberté individuelle et le droit à la sécurité publique.

La détention provisoire peut être ordonnée à tout stade de l'enquête de police ou des procédures pénales, ou à tout moment entre le début de l'enquête de police jusqu'à ce que la condamnation pénale soit devenue définitive, et il n’y a pas de date limite pour cette détention.

Comme la détention provisoire est une mesure extrême, il doit y avoir des indices sur l'auteur du crime ainsi que les preuves d'un crime (matérialité). En outre, elle ne peut être décrétée que dans les cas suivants prévus à l'article 312 du Code de procédure pénale: a) la garantie de l'ordre public (lorsque le séjour en liberté de l'accusé présente un danger significatif 
pour la société en raison de la probabilité établie qu'il va commettre d'autres crimes); b) assurer l'ordre économique (pour la préservation de l'ordre économique et la répression des crimes contre le système financier national); c) la garantie d'une enquête criminelle ${ }^{7}$ (si l'accusé en liberté fait obstacle à la recherche d'éléments d'information dans l'enquête de police); d) l'application de la loi pénale (veiller à ce que la prestation judiciaire pénale ne soit pas compromise); e) violation de mesure différente de celle de la détention (dans le cas où le condamné ne respecte pas quelques-unes des mesures de précaution différentes de celles de la détention précédemment accordée par le juge en sa faveur).

\section{PRINCIPALES RÉFORMES DANS LA PROCÉDURE PÉNALE BRÉSILIENNE}

Dans le cadre de la procédure pénale brésilienne, des changements importants ont eu lieu au cours des dernières années. L'un d'entre eux a été introduit par la loi 11.719 de 2008, qui a favorisé un changement substantiel dans les procédures. Comme l'intention du législateur était de faciliter la procédure, l'audience d'instruction et de jugement doit être tenue, en règle générale, en une seule séance, avec la concentration des actes de procédure (il peut être nécessaire, cependant, de démembrer l'audience compte tenu de la complexité de la cause). Ainsi, en règle générale, sont pratiquées dans une seule audience d'instruction et de jugement les étapes de procédure suivantes: collecte des déclarations de la victime, audition des témoins de l'accusation et de la défense, explications des experts, confrontations, reconnaissance des personnes et des choses, l'accusé étant alors interrogé. À cet égard, cette réforme a été un atout majeur pour les défenseurs, car ils seront en mesure de préparer la thèse de défense de leurs clients après la phase de production des preuves à l'audience d'instruction et de jugement. Autrement dit, au moment où l'accusé sera interrogé, il saura tout ce qui a été produit et mis en évidence à son sujet lors de l'audience.

\footnotetext{
${ }^{7}$ Pour Machado (2009, p. 463), l'instruction criminelle est utile lorsque, par exemple, l'auteur de l'acte criminel, en liberté, peut gêner la collecte des preuves, soit en faisant disparaître les vestiges qui composent le corpus delicti, soit en menaçant des témoins et des experts ou même en détruisant et rendant d'autres preuves inutilisables (documents, choses, etc.)
} 
Un autre changement important est survenu dans le jury. Selon la Constitution brésilienne, le jury a le pouvoir de juger les crimes intentionnels contre la vie, qui sont: l'assassinat, l'incitation, l'instigation ou l'assistance au suicide, l'infanticide et l'avortement. Quant à la procédure du jury, les modifications ont été introduites par la loi 11.689 de 2008. Parmi celles-ci, on peut citer: a) la réduction de l'âge minimum de 21 ans à 18 ans pour faire partie du jury; b) la possibilité d'acquittement de l'accusé par le juge, s'il a été prouvé: qu'il y a absence du fait; que l'accusé n'est pas l'auteur ni n'a participé au fait; que ce fait ne constitue pas une infraction pénale; s'il est démontré que c'est un cas d'exemption de peine ou d'exclusion du fait de crime. (Ce changement est significatif, étant donné qu'il a évité que l'accusé soit inutilement mis en jugement en séance plénière devant le jury); c) la suppression d'un recours propre à la défense, appelé « demande d'un nouveau jury », qui était admis dans la loi précédente, lorsque la peine imposée était égale ou de plus de 20 ans; d) la possibilité de mener à bien la défense, même sans la présence de l'accusé; e) l'interruption du vote lorsque qu'il y a plus de trois voix pour acquitter ou condamner l'accusé. (Comme le nombre de jurés au Brésil est de sept le législateur a reconnu qu'il n'était pas nécessaire de continuer à voter sur chaque élément jusqu'à la fin, ce qui a assuré la protection du Jury face à de possibles insatisfactions des accusés et de leurs familles, comme de même l'anonymat des composants de ce Jury).

La loi 12.403, 2011, telle que modifiée par la loi 13.257 de 2016 a également apporté des changements importants dans la procédure pénale brésilienne. L'un de ces changements concerne l'application du régime de l'assignation à domicile, prévue aux articles 317 et 318 du Code de procédure pénale, qui consiste dans la détention de l'accusé à sa résidence dans les cas suivants: a) lorsque la personne a plus de 80 ans; b) lorsqu'elle est extrêmement affaiblie par une maladie grave; c) lorsqu'elle est indispensable pour la prestation de soins spéciaux à une personne de moins de 6 ans d'âge ou handicapée; d) lorsqu'il s'agit d'une femme enceinte; e) lorsque c'est une femme avec un enfant de 12 ans ou moins; f) lorsque c'est un homme unique responsable de la garde d'un enfant de 12 ans ou moins. Notons que dans tous ces cas, pour l'application de l'assignation à domicile, le juge exigera une preuve des exigences établies dans cet article. 
Un autre changement introduit dans le Code de procédure pénale brésilienne apporté par la loi $12.403^{8}$ se réfère à l'institution de ce qu'on appelle les mesures de précaution différentes de celles de la détention ${ }^{9}$ qui doivent être appliquées en observant l'état de nécessité et la gravité du crime, les circonstances du fait et les conditions personnelles de l'accusé. Autrement dit, la loi prévoit des hypothèses qui profitent à l'accusé, empêchant qu'il soit arrêté sans que cela soit nécessaire. Les mesures de précaution suivantes différentes de celle de la détention ont été instituées: I - se présenter régulièrement devant le tribunal, dans les temps et dans des conditions imposées par le juge, pour communiquer et justifier ses activités; II interdiction d'avoir accès ou de fréquenter certains lieux; III - interdiction de rester en contact avec une certaine personne; IV - interdiction de s'absenter de la juridiction lorsque cette présence est convenable ou nécessaire à l'enquête; $\mathrm{V}$ - assignation à domicile la nuit et les jours de congé lorsqu'une personne objet d'une enquête ou accusée a une résidence permanente et un travail fixe; VI - suspension de l'exercice de la fonction publique ou d'une activité économique quand existe la crainte de leur utilisation pour la pratique d'infractions pénales; VII - détention provisoire de l'accusé dans les cas de crimes commis avec violence ou menace grave, lorsque les experts ont conclu qu'on pouvait les lui imputer ou alors partiellement et qu'il y avait un risque de réitération du crime; VIII - caution, pour les infractions qui l'admettent, pour assurer la comparution à des actes de la procédure, pour éviter de bloquer sa marche ou en cas de résistance injustifiée à une décision de justice; IX - surveillance électronique.

On remarque que les modifications introduites dans la loi de la procédure pénale brésilienne au cours des dernières années ont eu pour but d'ajuster le Code de procédure pénale, daté de 1941, à l'esprit des dispositions normatives de la Constitution fédérale de 1988. Cependant, de nouvelles propositions de changement législatif sont actuellement examinées et discutées par le Congrès national brésilien, suite au travail de nombreuses audiences publiques qui ont compté sur la présence de députés, de sénateurs et de nombreux juristes.

\footnotetext{
8 La loi 12.343/11 a établi une liste de mesures conservatoires, substitutives et diverses de celles de l'emprisonnement, qui peuvent même être ordonnées indépendamment de l'arrestation provisoire, ou même isolément ou cumulativement. (POLASTRI LIMA, 2014, p. 752)

${ }^{9}$ Celles-ci ont un caractère substitutif, c'est-à-dire qu'elles sont des alternatives à l'arrestation provisoire, réservant la détention préventive comme le dernier instrument à utiliser. (LOPES Jr., 2012, p. 853).
} 


\section{RÉFÉRENCES}

CAPEZ, Fernando. Curso de direito penal: parte geral. 16. ed. São Paulo: Saraiva, 2012.

COÊLHO, Yuri Carneiro. Curso de direito penal didático. São Paulo: Atlas, 2014.

CUNHA JÚNIOR, Dirley da. Curso de direito constitucional. Salvador: Juspodium, 2008.

ESTEFAM, André. Direito penal: parte geral. V. 1. São Paulo: Saraiva, 2010.

FREITAS, Eduardo Alves de; SOUZA, Lúcio Flávio. O Ministério Público e a sua posição na ordem constitucional de 1988. In: Revista do Ministério Público do Estado de Goiás. Goiânia: ESMP-GO, 2013.

GRECO, Rogério. Curso de direito penal: parte geral, V. 1. 16. ed. Niterói, RJ, 2014.

GUIMARÃES, Guilherme Francisco Alfredo Cintra. Advocacia de Estado, administração pública e democracia: a função da consultoria jurídica na formulação e execução de políticas públicas. In: Revista da AGU. Ano X, nº 30. Brasília-DF: 2011.

ISHIDA, Válter Kenji. Processo penal. São Paulo: Atlas, 2009.

LENZA, Pedro. Direito constitucional esquematizado. 11. ed. Sâo Paulo: Método, 2007.

LOPES Jr., Aury. Direito processual penal. 9. ed. São Paulo: Saraiva, 2012.

MACHADO, Antônio Alberto. Curso de processo penal. 2. ed. São Paulo: Atlas, 2009.

MIRABETE, Júlio Fabbrini. Processo penal. 18. ed. São Paulo: Atlas, 2007.

PACELLI, Eugênio; FISCHER, Douglas. Comentários ao código de processo penal e sua jurisprudência. 5. ed. São Paulo: Atlas, 2013.

OLIVEIRA, Tarsis Barreto. Pena e racionalidade. 3. ed. Rio de Janeiro: Lumen Juris, 2017.

POLASTRI LIMA, Marcellus. Curso de processo penal. 8. ed. Brasília: Gazeta Jurídica, 2014.

Submetido em: 01/07/2019

Aceito em: 22/07/2019 